\title{
An Integrated Bayesian-Markovian Framework for Ascertaining Cost of Executing Quality Improvement Programs in Manufacturing Industry
}

\begin{abstract}
Purpose: Typically, the budgetary requirements for executing a supplier's process quality improvement program are often done in unstructured ways in that quality improvement managers purely use their previous experiences and pertinent historical information. In this backdrop, the objective of this research is to ascertain the expected cost of carrying out suppliers' process quality improvement programs that are driven by original equipment manufacturers (OEMs).
\end{abstract}

Methodology: Using inputs from experts who had prior experience executing suppliers' quality improvement programs and employing the Bayesian theory, transition probabilities to various quality levels from an initial quality level is ascertained. Thereafter Markov chain concept enables us to determine steady state probabilities. These steady state probabilities in conjunction with quality level cost coefficients yields the expected cost of quality improvement programs.

Findings: The novel method devised in this research is a key contribution of our work. Further, various implication related to experts' inputs, dynamics related to Markov chain etc. are discussed. The method is illustrated using a real life of automotive industry in India.

Value: Our research contributes to the extant literature in that a new method of determining the expected cost of quality improvement is proposed. Further, our method would be of value to original equipment manufacturers and suppliers wherein the quality levels at a given time is function of quality levels in preceding period(s).

Keywords: Cost of Quality, Quality Improvement Program, Manufacturing Industry

http://mc.manuscriptcentral.com/ijgrm 


\section{Introduction}

The dynamics of manufacturing industry particularly product centric manufacturing has evolved from the era of mid to late twentieth century (Spring et al., 2017). Between the period of 1960s1990s OEMs (original equipment manufacturer) used to get themselves involved at all levels of value-chain in that OEM's role ranged from at one end of the spectrum as component manufacturer to another end as assembler. However, with increased competition amongst OEMs, OEMs were forced to focus on their core activities (e.g. assembling and selling automobiles in case of automobile industry) thereby outsourcing much of the important but auxiliary activities for example component manufacturing to suppliers (Ciravegna et al., 2013). Easier technology availability also played a pivotal role in ensuring that suppliers in manufacturing industry developed their technical expertise so as to meet the demands of OEMs both in terms of quality and volumes. Infact, in today's era be it automotive industry, construction equipment industry or any other product centric manufacturing, there are often very few critical items that OEMs manufacture in-house; rather they rely on suppliers possessing specific design and manufacturing expertise. A case in point is the example of Bosch that supplies fuel injection pumps (FIPs) for engines to major automotive OEMs across the world (Bosch Automotive Catalogue).

Specifications of the components/subsystems that suppliers supply to specific OEMs are of-course contingent upon OEMs' requirements, suppliers' technical capabilities, customer requirements and so forth. In order to ensure desired quality level of incoming aggregates, OEM often support the suppliers both from a technical and resource standpoint. Supplier quality improvement programs (SQIP) is one such institutionalized and collaborative mechanism wherein a particular OEM enables suppliers to institutionalize organizational wide continuous quality improvement program encompassing inter Italia manufacturing process improvements (Uluskan et al., 2016 \& Noshad et al., 2013).

One of the key decisions that OEMs need to figure out relates to the budget allocation related to carry out such kind of manufacturing process improvement programs at suppliers' facilities. Estimating the pertinent budget is still easier in case of already established technologies requiring minor upgrades. However, when it comes to predicting the costs associated with executing manufacturing process improvement programs for aggregates requiring newer technologies, it gets 
challenging and fuzzy in that the supplier/OEMs struggle to identify the current level of technical competencies that the supplier possesses and ascertain extent of inputs desired to bring the suppliers' competencies to an acceptable level (Khanna et al., 2014). Essentially in such situation, the costs associated with executing manufacturing process improvements program at suppliers end would be often non-deterministic in nature and would essentially be characterized by some measure of expected cost. Further, transition of a particular supplier from the standpoint of manufacturing competency cannot be often identified with complete certainty in that there would always be certain transitional probability associated. This implies that given there are for argument sake five different quality levels as far as manufacturing processes at a supplier is concerned namely: a) quality level with unacceptable level of defects (QLUL); b) quality level with major level of defects (QLMAL); c) quality level with moderate level of defects (QLMOL); d) quality level with minor level of defects (QLMIL); e) near perfect quality level $(Q N L)$. Assuming that a supplier's initial state is $Q L U L$, then supplier transitioning to higher quality levels viz. $Q L M A L$, $Q M O L, Q L M I L, Q L U L$ would be characterized by four different transition probabilities. Ofcourse any OEM would want its supplier(s) to transition to $Q N L$ irrespective of the starting quality level of the pertinent supplier (essentially transition probability to state $Q N L$ as one). However, in reality this is not the case, since this transition depends upon a number of factors such as ability of the supplier's top management to sustain with the improvement initiatives, coherence amongst team members in execution of process improvement program, assimilation of the technical dimensions related to process improvement initiatives by the workforce and so forth. Moreover, the costs associated with improving the quality level associated with manufacturing processes at a particular supplier's facility would be higher in case of say making the desired transition from "quality level with unacceptable defects" to "near perfect quality level" than desired transition from "quality level with moderate defects" to "near perfect quality level". For an OEM, ascertaining a reasonable estimate of cost of implementing manufacturing process improvement initiatives to be undertaken at a supplier's facility holds all the more importance due to the fact that these budgetary estimates serve as a key inputs to the tactical and strategic planning both for the supplier and the associated OEM (Sarkar et al., 2017).

Therefore, to this end in this research, following two key research questions are addressed. 
a) How can we evolve a pragmatic mathematical model yielding expected cost measure that can serve as a starting point to ascertain the cost that OEMs can earmark to carrying out process quality improvements at a supplier's facility?

b) How can we incorporate dimensions related to transition of a particular supplier's quality related capabilities in such as manner that such transitions occur in a probabilistic manner?

To respond to these two primary research questions, in this paper, we consider case of a typical automotive OEM aiming to ascertain the expected cost of carrying out the process quality improvement process (PQIP) at a supplier's facility. In context of the research problem that we are addressing in this paper, Figure 1 illustrates the broad schema of the research.

\section{$<<$ Insert Figure 1 here $>>$}

Referring to Figure 1, there are essentially two stakeholders within our research framework - the OEM and the supplier. The OEM drives the PQIPs at the supplier's facility. The supplier is assumed to have very limited capabilities from process quality standpoint. However, given the execution of PQIP at the supplier's premises, the initial quality level of the associated supplier transitions into different superior quality levels with certain transition probabilities. In order to ascertain these transition probabilities, we utilize pertinent expert's opinion in conjunction with Bayesian theory. Thereafter employing principles of Markovian chain, steady state probabilities of different quality levels are quantified. Finally, the expected cost of carrying out PQIP at the supplier's facility is determined.

Rest of the article is arranged as follows. Section 2 and Section 3 details literature review and problem setting respectively. Illustration using a real-life case is presented in Section 4. Section 5 presents the results and discussions. Section 6 presents the managerial implications. Finally, conclusions and future research direction is presented in Section 7.

\section{Literature review}

The problem that we are addressing through our research pertains to modeling for supplier quality management. Bayesian theory and Stochastic process - specifically Markov chain are other two important dimensions that provides inputs to the framework that we intend to develop. Against 
this backdrop we discuss some recent and relevant research literature. Although studies related to ascertaining the cost of quality including cost of executing process improvements are relatively sparse, the related literature presented in this research is further segregated in three broad categories viz. case-study based research, empirical investigation based, analytical \& theoretical studies.

\subsection{Empirical studies}

Uluskan et al. (2016) emphasized the role of quality cost in making outsourcing decisions. Specifically, employing a survey based approach and utilizing the linear discriminant analysis (LDA), this research illustrated that by instituting quality improvement programs focused at both enhancing productivity and reducing variability, OEMs dependent on such suppliers can enhance their competitiveness. Plewa et al., (2014) provided the empirical evidence concerning the central role of quality related costs in a prevention-appraisal-failure model of quality in context of modern manufacturing. Specifically, using a regression based methodology it was concluded that substantial savings in cost of quality is possible when suppliers reach towards overall higher overall quality levels. O'Neill et al., (2016) integrated the quality financial performance paradigm into the operational performance metrics for Australian manufacturing firms thus contributing to the extant literature in that quality orientations were developed to evaluate various financial performance outcomes. A key contribution of the study pertained to the fact that quality orientation differentiates financial performance. An accompanying limitation however pertained to limited generalizability to wider manufacturing, service and international contexts.

\subsection{Case-study based research}

Tye et al., (2011) explored the cost of quality implementation and related effects on manufacturing firms in Malaysia. Using a case study and interview based approach it was indicated that costs incurred in implementing quality at manufacturing firms aid in mitigating other costs such as customer complaints, rework, warranty expenditure etc. However, a key imitation pertained to the application of subjective method of ascertaining the cost of quality. Kaipia et al., (2017) investigated the influence of quality priorities in managing integration in outsourcing relationships. 
Using a case based method, the effects of quality and cost on how different modes of integration are used both before and in early phases of production in context of Food and Electronics manufacturing organization were addressed. The quality priority comprised of the relationships where the main investment and emphasis were directed towards ensuring the supplier's ability to provide reliable inputs. Chiarini et al., (2015) investigated the differences in terms of the effects of non-conformity processes on cost of quality in capital intensive manufacturing companies. Using the case of 42 companies and employing an administered questionnaire based method, the study illustrated that there is no difference within and between the manufacturing sector in terms of cost of poor quality. Chopra et al., (2015) employing a case of small and medium size enterprise (SME) working in automotive domain in conjunction with regression analysis determined that total failure cost has a direct positive correlation with total quality costing in that this correlation increases with time.

\subsection{Analytical studies}

Alglawe et al., (2017) in their study of ascertaining cost of quality used system dynamics approach. A key contribution of the study was that it examined the effects of opportunity costs in quality costing calculations. Another important contribution of the study pertained to successful integration of prevention-appraisal-failure concept, quality levels, and opportunity costs into quality costing. Omar et al., (2014) in their study of ascertaining the cost of quality devised an improved mathematical model using real-life industrial data. The evolved simulation based model had a number of merits with respect to previous models viz. ability to capture variability in costing and better experimentation. A key finding pertained to the fact that reduction in failure costs can be achieved at low level of increase in conformance expenditures. Ramudhin et al., (2008) devised a mathematical programming based analytical model to incorporate cost of quality in the supply chain network. This model was of significant value to industries (such as aerospace) where variable production costs are high, hence producing extra parts to compensate for defects would be a costly option. The research was infact a one of the few work that successfully integrated cost of poor quality into the paradigm of supply chain modeling. Kang et al., (2018) devised a multi-objective and discrete event simulation based framework to model the process improvement implementation framework. Specifically, within the related research framework, reduction in lead time and 
rationalization of total inventory holding cost were modelled as surrogate measure of process improvement, thus contributing to the extant literature in terms of a methodological contribution to the research literature pertaining to continuous process improvement (CPI).

Table 1 presents the taxonomy of the literature review contrasting key research dimensions with respect to the research we have carried out in this work.

$$
<<\text { Insert Table } 1 \text { here }>>
$$

\section{Problem setting}

\section{Indices}

\begin{tabular}{|l|l|}
\hline Notations & Description \\
\hline$i=(1,2, \ldots n)$ & Indices for state of process quality level for supplier. \\
\hline$m=(1,2, \ldots M)$ & Indices for decision maker belonging to the OEM. \\
\hline$j$ & Index for absorbing state of quality level for supplier. \\
\hline
\end{tabular}

\section{Parameters}

\begin{tabular}{|c|c|}
\hline Notations & Description \\
\hline$r^{m}\left(i \rightarrow i^{*}\right)$ & Pairwise comparison value of state " $i$ " with respect to state " $i$ "” by expert " $m$ " \\
\hline$X\left(i \rightarrow i^{*}\right)$ & $\begin{array}{l}\text { Random variable associated with transition probability from state " } i \text { " to state } \\
\text { " } i " \text { ". }\end{array}$ \\
\hline$p^{m}\left(i \rightarrow i^{*}\right)$ & $\begin{array}{l}\text { Based on the input of expert " } m \text { ", transition probability value from state " } i \text { " to } \\
\text { state " } i * \text { ". }\end{array}$ \\
\hline$p\left(i \rightarrow i^{*}\right)$ & $\begin{array}{l}\text { Mean scaled probability value of transition probability from state " } i \text { " to state } \\
\text { " } i * \text { " such that } i \neq i \text {. }\end{array}$ \\
\hline$\pi(i)$ & Mean value of the steady-state probability corresponding to the state " $i$ ". \\
\hline$C(i)$ & SQIP's associated cost corresponding to state " $i$ ". \\
\hline
\end{tabular}

The need for a critical assessment of the quality improvement programs in terms of accompanying efficacy and effectiveness has been aptly discussed in the study of Antony et al., (2016). This study 
also emphasized that quality improvement programs be viewed from a resource-based perspective as well such that appropriate trade-offs can be established between cost and quality. Therefore, in this backdrop, within our problem environment, an OEM need to ascertain the expected cost that it would have to bear to support the process quality improvement initiatives at a particular supplier's facility. In the backdrop of the OEM supporting the supplier for implementation of new technology at the supplier's facility, we assume that the initial state of process quality level at supplier's facility is $Q L U L$. If the underlying problem is modelled along stochastic process (more specifically Markov chain), $Q L U L$ (initial state) can transition to different states i.e. $Q L M A L$, QLMOL, $Q L M I L$, and $Q N L$ with associated transition probabilities. The rationale for modeling the problem along the Markov chain is grounded essentially in the fact that transition probabilities involving how the process improvement program will evolve in the future would depend only on the current state of the Markovian process. Since the process improvement program at the supplier's facility would be driven by the OEM's prior experiences in executing similar PQIP with other suppliers, we model the problem in such a way that there would be " $m$ " number of subject matter experts (on the basis of their prior technical experiences executing similar programs) would provide inputs in ascertaining the initial transition probabilities. Since these transition probabilities cannot be ascertained directly and also to mitigate subjectivities, we employ the pair-wise comparison and subsequent Bayes theory-based probability determination approach to ascertain the transition probabilities (Chin et al., 2009 and Goswami, 2018).

\subsection{Determination of transition probabilities}

Let there exists $I$ different states corresponding to respective process quality levels i.e. 1,2 , $3 \ldots i \ldots n$. The transition probability from state " $i$ " to state " $i$ " as obtained based on inputs from member " $m$ " i.e. $p^{m}\left(i \rightarrow i^{*}\right)$ need to be ascertained. $p^{m}\left(i \rightarrow i^{*}\right)$ can be estimated (some kind of point estimate) directly from operational managers within the OEM possessing prior experience in implementation of PQIPs. However, such approach will be recommended in case of lower number of states in that with higher number of states corresponding to respective quality levels, directly estimating transition probabilities for all the states may invariably contain biases and inconsistencies. Alternatively, a more accurate evaluation can be carried out by comparing just 
two states for two corresponding quality levels (rather than $n$ different states) employing the pairwise comparison matrix presented in Table 2(a).

$<<$ Insert Table 2 here $>>$

Referring to Table 2, the element, $r^{m}\left(i \rightarrow i^{*}\right)$ can be derived by responding to question such as "comparing the state corresponding to quality level $i$ with that corresponding to quality level $n$, which one is more likely to happen and how much more/less likely?".

Therefore, we would have $n^{2}$ number of comparisons in the matrix presented in Table 2(a). The relatively priority of state corresponding to a particular quality level " $i$ " can be determined employing maximum eigenvector $\mu$, where $\mu$ would be expressed using the following mathematical expression.

$$
\mu=\left[\mu_{1}, \mu_{2}, \mu_{3}, . . \mu_{i} . . \mu_{k} . . \mu_{n}\right]^{T}
$$

A comparison matrix with $\mathrm{CR}$ (consistency ration) less than 0.1 would be considered unacceptable. As the sum of all the elements in $\mu$ equals to 1 and its $i^{\text {th }}$ element $-\mu_{i}$ denotes the relative importance of state corresponding to quality level " $i$ ", it would be obvious to interpret $\mu_{i}$ as the transition probability of the state corresponding to quality level " $i$ " such that the following mathematical relationship is satisfied.

$p^{m}\left(i \rightarrow i^{*}\right)=\mu_{i}$

Essentially, transition probability determination is grounded in the Bayesian theory (Chin et al., 2009).

\subsection{Modeling the problem along Markov chain}

When modeling the said problem along Markov chain, an implicit assumption we make that the states corresponding to respect quality levels follows a discrete Markov chain following a stochastic process in that $\left\{X_{i}, \mathrm{i}=0,1,3 \ldots \ldots\right\}$ such that $X_{i}$ is the quality level corresponding to quality level " $i$ ". The Markovian property considered in this research corresponds to the 
improvement process as opposed to decay process (such as birth-and-death process or machine deterioration due to continuous production).

If there are $M$ different subject matter experts giving their inputs in ascertaining the transition probabilities, then mean transition probability " $p\left(i \rightarrow i^{*}\right)$ " would be function of mean transition probabilities $p^{m}\left(i \rightarrow i^{*}\right)$ as ascertained by different experts in that the following mathematical relationship would be satisfied.

$$
p\left(i \rightarrow i^{*}\right)=\sum_{m \in M} \frac{p^{m}\left(i \rightarrow i^{*}\right)}{M} \quad \forall i \text { and } i \neq i^{*}
$$

Further, the steady state probabilities " $\pi(i)$ "s corresponding to the state of quality level " $i$ " can be expressed in terms of " $p\left(i \rightarrow i^{*}\right)$ "s as per the following mathematical expression.

$$
\pi(i)=p(i \rightarrow 1) \cdot \pi(1)+p(i \rightarrow 2) \cdot \pi(2)+\ldots \ldots \ldots p\left(i \rightarrow i^{*}\right) \cdot \pi\left(i^{*}\right)+\ldots \ldots . p(i \rightarrow I) \cdot \pi(I) \quad \forall i .
$$

Further, sum of steady state probabilities must be equal to 1 . This is represented using the following equation.

$$
\sum_{i \in M} \pi_{i}=1
$$

Solving simultaneous equations corresponding to equation 5 and 6 would yield the values of all the steady state probabilities i.e. $\pi(1), \pi(2), \ldots \ldots \ldots \pi(i) \ldots \ldots . . \pi(I)$.

Once we have all the steady state probability, the expected cost of executing the SQIPs would be determined as equal to $\sum_{i \in I} C(i) \cdot \pi(i)$.

\section{Illustration using a real-life case}

We in this research consider a case of automotive industry in India in that we consider an example of India's premier automobile company whose managerial leaders have had significant number of man-years driving the supplier quality improvement programs at different types of suppliers be it component focused, sub-assembly focused or engineering system focused. Most of the supplier's 
facilities where the improvement programs were executed have their own inhouse design and manufacturing capabilities as opposed to purely possessing only manufacturing capabilities.

Now in order to demonstrate the evolved framework, we take inputs from 30 different experts (primarily at the middle to senior management level) from the case organization (automotive OEM) who had primarily executed PQIPs are at sheet metal components' suppliers' facilities. The steps for demonstrating the evolved framework for the real-life example is illustrated in Figure 2.

$<<$ Insert Figure 2 here $>>$

The steps as detailed in Figure 2 is illustrated in detail as per the following sections.

4.1 Ascertain the quality levels and populate pairwise component matrices

The illustration starts with ascertaining the supplier quality levels and population of pairwise comparison matrices (based on inputs from 30 different experts). The initial state of the quality level is assumed to be $Q L U L$. A total of five different states corresponding to five respective quality levels are considered in this research. These levels are tabulated in Table 3(a).

$<<$ Insert Table 3 here $>>$

Referring to Table 3(a), it is to be noted that the initial state $i=1$ corresponds to the unacceptable quality level. Since, in this research we are trying to ascertain the expected budget when implementing PQIP at a particular supplier's facility in context of the new technology, we inherently assume that $Q L U L$ would be the initial state. From a Markovian perspective, this initial state in the improvement process correspond to the absorbing state in the decay process.

Based on the inputs from all 30 experts the pair-wise comparison is populated. For the brevity of illustration, inputs from $1^{\text {st }}$ expert is only enlisted in Table 3(b). Referring to Table 3(b), values of $r^{1}(1 \rightarrow 1), r^{1}(1 \rightarrow 2), r^{1}(1 \rightarrow 3), r^{1}(1 \rightarrow 4)$, and $r^{1}(1 \rightarrow 5)$ are 3/2, 2, 4, 3/2, 1 respectively. This implies that the first expert's assessment of transitions i.e. "QLUL to QLMAL", "QLUL to QLMOL", and "QLUL to $Q L M I L "$ is 2 times, 4 times, and 1.5 times respectively that of supplier's quality level transitioning from $Q L U L$ to $Q N L$. Also, supplier's quality level stuck at QLUL is 1.5 times that of transitioning from $Q L U L$ to $Q N L$. It is to be further noted that a particular quality level will not deteriorate. This means that from example QLMIL will not transition to QLUL, QLMAL, QLMOL. Therefore, such comparison ratings are set as zero. 
4.2 Determination transition probabilities

As far as determining the transition probabilities are concerned, we utilize the approach as discussed in Section 3.1. The resulting transition probabilities based on the inputs from the $1^{\text {st }}$ expert is illustrated in Table 4(a).

$$
<<\text { Insert Table } 4 \text { here }>>
$$

Referring to Table 4(a), it to be noted that sum of probabilities across the rows would be equal to one. For instance, referring to row 1 , sum of $0.417,0.278$. $0.162,0.074$, and 0.070 would be equal to one.

Based on the inputs and subsequent determination of transition probability from remaining 30 experts, we also tabulate the values for mean transition probabilities that are listed in Table 4(b). These values are scaled in such a manner that sum of transition probabilities add up to one for all rows.

\subsection{Ascertain steady state probabilities}

The steady state probabilities that we are trying to ascertain in this section essentially refer to the mean steady state probabilities. The mean steady state probabilities $\pi(1), \pi(2), \pi(3), \pi(4)$, and $\pi(5)$ refer to quality levels $Q L U L, Q L M A L, Q L M O L, Q L M I L$, and $Q N L$ respectively. Using equation 4 and utilizing the inputs from Table 4(b), following set of simultaneous equation can be populated.

$$
\begin{aligned}
& \pi_{1}=\left(0.398 \cdot \pi_{1}+0.319 \cdot \pi_{2}+0.128 \cdot \pi_{3}+0.089 \cdot \pi_{4}+0.066 \cdot \pi_{5}\right) \\
& \pi_{2}=\left(0.269 \cdot \pi_{2}+0.327 \cdot \pi_{3}+0.198 \cdot \pi_{4}+0.206 \cdot \pi_{5}\right) \\
& \pi_{3}=\left(0.249 \cdot \pi_{3}+0.248 \cdot \pi_{4}+0.504 \cdot \pi_{5}\right) \\
& \pi_{4}=\left(0.68 \cdot \pi_{4}+0.32 \cdot \pi_{5}\right)
\end{aligned}
$$

Using equation 5, following equation can be written. 
$\pi_{1}+\pi_{2}+\pi_{3}+\pi_{4}+\pi_{5}=1$

Solving equation 6 to 10 results in the following mean steady state probabilities.

$\left\{\pi_{1}, \pi_{2}, \pi_{3}, \pi_{4}, \pi_{5}=0.199,0.208,0.221,0.092,0.28\right\}$

\subsection{Determine process improvement cost}

In order to determine the expected process improvement cost, the first task is to ascertain a tentative estimate of the process improvement cost that would be required to: a) improve the process quality from inferior to superior level; b) sustain the quality level. Essentially, when we talk about this cost coefficient, it essentially represents in surrogate manner dimensions related to the cost of quality i.e. prevention, appraisal, internal failure cost, external failure cost and so on. Based on the inputs from all the experts, we tabulate a tentative estimate of the process improvement cost coefficients. These values are listed in Table 5.

$<<$ Insert Table 5 here $>>$

Based on these cost coefficients and values obtained corresponding to the steady state probability values in conjunction with equation 6 , the expected cost of the carrying out the process improvement at the given supplier is ascertained as $\$ 205,405$.

\section{Results and discussions}

Referring to the steady state probability values, it can be ascertained that based on qualitative inputs from experts and using the quantitative analysis grounded in Bayesian theory and Markov chain, the most likely quality level that the supplier would be transitioning from process quality standpoint is near perfect level of quality $(Q N L)$. However, inspecting the associated steady state value corresponding to this quality level also establishes that probability of transitioning to this quality (from early quality level) is not very dominant vis-à-vis transitioning to other quality levels. Further, even after executing the PQIP at the supplier's facility, there is around $20 \%$ chance that supplier's process quality level might not improve. We sought to rationalize this aberration in that most likely steady state quality level is $Q N L$, while at the same time significant chances are that 
supplier process quality level would be rather the lowest level. One of the possible justifications for this anomaly is that the particular supplier had rather heterogenous mix of technical workforce in that certain fraction of workforce were technically very proficient; while on the other hand other chunk of workforce were technically relatively deficient as well as unmotivated. This heterogeneity however got reflected in the supplier's capability assessment by experts in that few expert's judgment would have been dominated by share of technical workforce having high proficiency level; on the other hand, few expert's judgement would have been dominated by share of technical workforce possessing lower proficiency level and/or motivation. However, this varied judgement would be mitigated to an extent since mean transition probabilities are being considered in our devised model.

One of the methodological contributions related to our work is that unlike vast majority of the extant research literature that assumes that a supplier's process quality level would improve deterministically into higher quality levels, we model the problem of estimating the expected cost of process quality improvement in such a manner that process quality would transition to higher levels probabilistically. However, this probabilistic transition is also a function of previous quality level(s) - an important consideration associated with Markov chain. This probabilistic transition from one quality level to superior quality level(s) also given a crucial input to top management of both OEM and supplier in that the expectations resulting from implementing process quality improvement programs can also be aligned to structured analysis as opposed to fixing these expectations in isolation.

As far as determining the expected cost of process improvement quality is concerned, in this research we have considered a point based estimate resulting in a single expected cost value. However, employing fuzzy values from the experts could have also determined a tentative optimistic and pessimistic values of expected cost of process improvement within which actual expected value would lie. For the sake of illustration in this research, we have considered only five different quality levels for the sheet metal component supplier. Since from a technological standpoint, a sheet metal component supplier's capabilities are rather easier to classify in different discrete buckets; however, for other suppliers related to rather complex subsystems such a fuel injection pumps etc., the number of discrete process quality levels would be much higher. Owing to this fact for such type of suppliers a more computationally intensive effort would be required. 


\section{Managerial implications}

For an OEM, continuous improvement in its and associated suppliers' technical and process capabilities are an important tactical decision for which OEMs often need to earmark significant budgetary provisions out of the annual anticipated operational costs. Anecdotally speaking, except for a few large global OEMs, most OEMs often take the decision pertaining to the level of expected budget for supplier process quality improvement in a rather unscientific and unstructured manner. Typically, quality improvement managers either rely on historical data or on their own empirical judgment for ascertaining the expected budget for executing the quality improvement programs. A key limitation of such unscientific and unstructured approach is that managers often end up overestimating or underestimating the expected cost associated with carrying out such programs at suppliers' facilities. A key feature of our evolved Bayesian-Markovian anchored framework in this research is that by adopting our framework, OEMs can minimize deviations either considering overestimation or underestimation. From a practitioner's perspective, our study augments the extant literature in a number of ways. By incorporating a Bayesian based pair-wise comparison grounded approach, we are able to ascertain relatively accurately various transition probabilities as far as different quality levels are concerned. This is a significant departure from often unstructured ways in which managers ascertain the quality levels associated with suppliers. Further, this approach also negates the presumption that after carrying out certain quality improvement program, the process quality level would automatically improve to certain other higher quality level in a sure shot manner. Further, our approach is rather unconvoluted and pragmatic in that once all possible quality levels are identified, pair-wise comparisons from all experts are ascertained, cost coefficients are estimated, solving a number of simultaneous equations fetches us the steady state probabilities associated with the respective quality levels. These steady state probabilities in conjunction with the cost coefficients associated with respective quality levels ultimately yields the expected cost of carrying out the quality improvement programs.

\section{Conclusion and future research direction}

In this research, we have undertaken an important problem of ascertaining the expected cost of carrying out suppliers' process quality improvement programs that are driven by OEM. The 
mathematical framework devised in this research is based on the Bayesian theory and Markov chain.

The starting point of the framework is identification of various quality levels that the supplier would transition to. The basic assumption in our research is related to the fact that the supplier's quality level is rather unacceptable at the beginning of the quality improvement program. Thereafter, employing the inputs from experts belonging to OEMs who had prior experience executing process quality improvement programs, pair-wise comparisons (contracting different quality levels) are carried out. These pair-wise comparisons enable us to ascertain the mean transition probabilities associated with different quality levels. Employing the Markovian theory, the implicit and realistic consideration of supplier's higher process quality levels vis-à-vis. function of lower quality level(s) is integrated in our framework. Once the mean transition probabilities are ascertained, simultaneous equations linking transition and steady state probabilities corresponding to respective quality levels are derived. Solving these simultaneous equations yield steady state probabilities associated with corresponding quality levels. The steady state probabilities in conjunction with associated cost coefficients of various quality levels results in obtaining the expected cost of executing the quality improvement program at the supplier's facility. The framework has been demonstrated using a real-life case from automotive industry in India.

There are however a few limitations of our devised framework. We have assumed the experts to have a uniform orientation as far as providing the inputs for transition probabilities are concerned. However, integration of behavioral dimensions of decision-making in terms of optimistic/pessimistic scenarios would further add value to the devised framework. The cost coefficients that we have ascertained from the experts can also be refined using appropriate statistical models wherein cost coefficients can be modelled in terms of dependent variable and other pertinent variables can modelled in terms of independent variable. 


\section{References}

Alglawe, A., Schiffauerova, A., and Kuzgunkaya, O., 2017, “Analysing the cost of quality within a supply chain using system dynamics approach", Total Quality Management and Business Excellence, DOI: 10.1080/14783363.2017.1400376.

Antony, J., and Karaminas, H., 2016, "Critical assessment on the Six Sigma Black Belt roles/responsibilities, skills and training”, International Journal of Quality and Reliability Management, 33(5), 558-573.

Chiarini, A., 2015, "Effect of ISO 9001 non-conformity process on cost of poor quality in capital intensive sectors", International Journal of Quality and Reliability Management, 32(2), 144-155.

Chin, K., S., Tang, D., W., Yang, J., B., Wong, S., Y., and Wang, H., 2009, “Assessing new product development project risk by Bayesian network with a systematic probability generation methodology", Expert Systems with Applications, 36(6), 9879-9890.

Cirvegna, L., Romano, P., and Pilkington, A., 2013, "Outsourcing practices in automotive supply networks: an exploratory study of full service vehicle suppliers", International Journal of Production Research, 51(8), 2478-2490.

Goswami, M., 2018, "Supply Chain Centric Product Line Selection: A Functional Risk Focused Approach", International Journal of Production Research, DOI: https://doi.org/10.1080/00207543.2018.1436788.

Kang, P., S., and Bhatti, R., S., 2018, “"Continuous process improvement implementation framework using multi-objective genetic algorithms and discrete event simulation", Business Process Management Journal, DOI: https://doi.org/10.1108/BPMJ-07-2017-0188.

Kapia, R., and Turkulainen, V., 2017, "Managing integration in outsourcing relationships — The influence of cost and quality priorities”, Industrial Market Management, 61(2017), 114129. 
Khanna, V., K., Gupta, R., 2014, “Comparative study of the impact of competency-based training on 5 "S" and TQM: a case study", International Journal of Quality and Reliability Management, 31(3), 238-260.

Noshad, K., and Awasthi, A., 2015, "Supplier quality development: A review of literature and industry practices”, International Journal of Production Research, 53(2), 466-487.

O’Neill, P., Sohal, A,., “Quality management approaches and their impact on firms' financial performance - An Australian study", International Journal of Production Economics, 171(3), 381-393.

Omar, M., K., and Murgan, S., 2014, ""An improved model for the cost of quality", International Journal of Quality \& Reliability Management” 31(4), 395-418.

Ozkan, S., and Karaibrahimoglu, Y., Z., 2013, ““'Activity-based costing approach in the measurement of cost of quality in SMEs: a case study", Total Quality Management \& Business Excellence, 24(3-4), 420-431.

Plewa, M., Kaiser, G., Hartmann, E., 2016, “Is quality still free?: Empirical evidence on quality cost in modern manufacturing", International Journal of Quality and Reliability Management, 33(9), 1270-1285.

Ramudhin, A., Alzaman, C., and Bulgak, A., 2008, "Incorporating the cost of quality in supply chain design", Journal of Quality in Maintenance Engineering, 14(1), 71-86.

Retrieved from http://www.bosch-automotive-catalog.com/en/vehicle-search.

Sarkar, B., Majumder, A., Sarkar, M., Dey, B., K., and Roy, G., 2017, “Two-echelon supply chain model with manufacturing quality improvement and setup cost reduction", Journal of Industrial and Management Optimization, 13(12), 1085-1104.

Spring, M., Hughes, A., Mason, K., \& McCaffrey, P., 2017, "Creating the competitive edge: A new relationship between operations management and industrial policy", Journal of Operations Management, 49-51(2017), 6-19. 
Tye, L., H., Halim, H., A., and Ramayah, T., 2011, “An exploratory study on cost of quality implementation in Malaysia: The case of Penang manufacturing firms", Total Quality Management \& Business Excellence, 22(12), 1299-1315.

Uluskan, M., Joines, J., A., and Godfrey, A., B., 2016, “"Comprehensive insight into supplier quality and the impact of quality strategies of suppliers on outsourcing decisions", Supply Chain Management: An International Journal, 21(1), 92-102. 
Table 1: Taxonomy of literature review 
Table 2: Transition ratings and probabilities

Table 2(a): Pairwise comparison ratings

\begin{tabular}{|c|c|c|c|c|c|c|c|}
\hline & \multicolumn{7}{|c|}{ States corresponding to respective quality levels } \\
\hline \multirow{2}{*}{$\begin{array}{c}\text { States } \\
\text { corresponding } \\
\text { to respective } \\
\text { quality levels }\end{array}$} & 1 & 1 & 2 &.. & $i$ & $\ldots$ & $n$ \\
\cline { 2 - 8 } & 2 & $r^{m}(1 \rightarrow 1)$ & $r^{m}(1 \rightarrow 2)$ & $r^{m}(1 \rightarrow .)$. & $r^{m}(1 \rightarrow i)$ & $r^{m}(1 \rightarrow i \ldots)$ & $r^{m}(1 \rightarrow n)$ \\
\cline { 2 - 8 } &.. & $r^{m}(. . \rightarrow 1)$ & $r^{m}(. . \rightarrow 2)$ & $r^{m}(. . \rightarrow 3)$ & $r^{m}(. . \rightarrow i)$ & $r^{m}(. . \rightarrow \ldots)$ & $r^{m}(. . \rightarrow n)$ \\
\cline { 2 - 8 } & $i$ & $r^{m}(i \rightarrow 1)$ & $r^{m}(i \rightarrow 2)$ & $r^{m}(i \rightarrow .)$. & $r^{m}(i \rightarrow i)$ & $r^{m}(i \rightarrow \ldots)$ & $r^{m}(i \rightarrow n)$ \\
\cline { 2 - 8 } & $\ldots$ & $r^{m}(\ldots \rightarrow 1)$ & $r^{m}(\ldots \rightarrow 2)$ & $r^{m}(\ldots \rightarrow .)$. & $r^{m}(\ldots \rightarrow i)$ & $r^{m}(\ldots \rightarrow \ldots)$ & $r^{m}(\ldots \rightarrow n)$ \\
\cline { 2 - 8 } & $n$ & $r^{m}(n \rightarrow 1)$ & $r^{m}(n \rightarrow 2)$ & $r^{m}(n \rightarrow .)$. & $r^{m}(n \rightarrow i)$ & $r^{m}(n \rightarrow \ldots)$ & $r^{m}(n \rightarrow n)$ \\
\hline
\end{tabular}

Table 2(b): Transition probabilities

\begin{tabular}{|c|c|c|c|c|c|c|c|}
\hline & \multicolumn{7}{|c|}{ States corresponding to respective quality levels } \\
\hline \multirow{7}{*}{$\begin{array}{c}\text { States } \\
\text { corresponding } \\
\text { to respective } \\
\text { quality levels }\end{array}$} & & 1 & 2 & .. & $i$ & $\ldots$ & $n$ \\
\hline & 1 & $p^{m}(1 \rightarrow 1)$ & $p^{m}(1 \rightarrow 2)$ & $p^{m}(1 \rightarrow .)$. & $p^{m}(1 \rightarrow i)$ & $p^{m}(1 \rightarrow i \ldots$ & $p^{m}(1 \rightarrow n)$ \\
\hline & 2 & $p^{m}(2 \rightarrow 1)$ & $p^{m}(2 \rightarrow 2)$ & $p^{m}(2 \rightarrow .)$. & $p^{m}(2 \rightarrow i)$ & $p^{m}(2 \rightarrow \ldots)$ & $p^{m}(2 \rightarrow n)$ \\
\hline & .. & $p^{m}(. . \rightarrow 1)$ & $p^{m}(. . \rightarrow 2)$ & $p^{m}(. . \rightarrow 3)$ & $p^{m}(. . \rightarrow i)$ & $p^{m}(. . \rightarrow \ldots)$ & $p^{m}(. . \rightarrow n)$ \\
\hline & $i$ & $p^{m}(i \rightarrow 1)$ & $p^{m}(i \rightarrow 2)$ & $p^{m}(i \rightarrow .)$. & $p^{m}(i \rightarrow i)$ & $p^{m}(i \rightarrow \ldots)$ & $p^{m}(i \rightarrow n)$ \\
\hline & $\ldots$ & $p^{m}(\ldots \rightarrow 1)$ & $p^{m}(\ldots \rightarrow 2)$ & $p^{m}(\ldots \rightarrow .)$. & $p^{m}(\ldots \rightarrow i)$ & $p^{m}(\ldots \rightarrow \ldots)$ & $p^{m}(\ldots \rightarrow n)$ \\
\hline & $n$ & $p^{m}(n \rightarrow 1)$ & $p^{m}(n \rightarrow 2)$ & $p^{m}(n \rightarrow .)$. & $p^{m}(n \rightarrow i)$ & $p^{m}(n \rightarrow \ldots)$ & $p^{m}(n \rightarrow n)$ \\
\hline
\end{tabular}

Table 3: Quality level and pair-wise comparison matrix 
Table 3(a): States corresponding to respective quality levels

\begin{tabular}{|c|c|c|}
\hline $\begin{array}{c}\text { States of quality } \\
\text { level }(i)\end{array}$ & Abbreviation & Description \\
\hline 1 & QLUL & Quality level with unacceptable level of defects \\
\hline 2 & QLMAL & Quality level with major level of defects \\
\hline 3 & QLMOL & Quality level with moderate level of defects \\
\hline 4 & QLMIL & Quality level with minor level of defects \\
\hline 5 & QNL & Near perfect quality level \\
\hline
\end{tabular}

Table 3(b): Pair-wise inputs from $1^{\text {st }}$ expert

\begin{tabular}{|c|c|c|c|c|c|c|}
\hline & \multicolumn{6}{|c|}{ States corresponding to respective quality levels $(i)$} \\
\hline \multirow{5}{*}{$\begin{array}{l}\text { States } \\
\text { corresponding } \\
\text { to respective } \\
\text { quality levels }\end{array}$} & & 1 & 2 & 3 & 4 & 5 \\
\hline & 1 & $r^{1}(1 \rightarrow 1)=3 / 2$ & $r^{1}(1 \rightarrow 2)=2$ & $r^{1}(1 \rightarrow 3)=4$ & $r^{1}(1 \rightarrow 4)=3 / 2$ & $r^{1}(1 \rightarrow 5)=1$ \\
\hline & 2 & $r^{1}(2 \rightarrow 1)=0$ & $r^{1}(2 \rightarrow 2)=1$ & $r^{1}(2 \rightarrow 3)=5$ & $r^{1}(2 \rightarrow 4)=5 / 2$ & $r^{1}(2 \rightarrow 5)=3 / 2$ \\
\hline & 3 & $r^{3}(3 \rightarrow 1)=0$ & $r^{1}(3 \rightarrow 2)=0$ & $r^{1}(3 \rightarrow 3)=4 / 3$ & $r^{1}(3 \rightarrow 4)=1$ & $r^{1}(3 \rightarrow 5)=3 / 2$ \\
\hline & 4 & $r^{1}(4 \rightarrow 1)=0$ & $r^{1}(4 \rightarrow 2)=0$ & $r^{1}(4 \rightarrow 3)=0$ & $r^{1}(4 \rightarrow 4)=7 / 2$ & $r^{m}(4 \rightarrow 5)=1$ \\
\hline (l) & 5 & $r^{1}(5 \rightarrow 1)=0$ & $r^{1}(5 \rightarrow 2)=0$ & $r^{m}(5 \rightarrow 3)=0$ & $r^{m}(5 \rightarrow 4)=0$ & $r^{m}(5 \rightarrow 5)=1$ \\
\hline
\end{tabular}

Table 4: Transition probabilities 
Table 4(a): Transition probabilities as determined for $1^{\text {st }}$ member

\begin{tabular}{|c|c|c|c|c|c|c|}
\hline & \multicolumn{6}{|c|}{ States corresponding to respective quality levels $(i)$} \\
\hline \multirow{6}{*}{$\begin{array}{c}\text { States } \\
\text { corresponding } \\
\text { to respective } \\
\text { quality levels } \\
\text { (i) }\end{array}$} & & 1 & 2 & 3 & 4 & 5 \\
\hline & 1 & $\begin{array}{l}p^{1}(1 \rightarrow 1) \\
=0.417\end{array}$ & $\begin{array}{l}p^{1}(1 \rightarrow 2) \\
=0.278\end{array}$ & $\begin{array}{l}p^{1}(1 \rightarrow 3) \\
=0.162\end{array}$ & $\begin{array}{l}p^{1}(1 \rightarrow 4) \\
=0.074\end{array}$ & $\begin{array}{l}p^{1}(1 \rightarrow 5) \\
=0.070\end{array}$ \\
\hline & 2 & $\begin{array}{l}p^{1}(2 \rightarrow 1) \\
=0\end{array}$ & $\begin{array}{l}r^{1}(2 \rightarrow 2) \\
=0.245\end{array}$ & $\begin{array}{l}r^{1}(2 \rightarrow 3) \\
=0.356\end{array}$ & $\begin{array}{l}r^{1}(2 \rightarrow 4) \\
=0.216\end{array}$ & $\begin{array}{l}r^{1}(2 \rightarrow 5) \\
=0.184\end{array}$ \\
\hline & 3 & $\begin{array}{l}r^{3}(3 \rightarrow 1) \\
=0\end{array}$ & $\begin{array}{l}r^{1}(3 \rightarrow 2) \\
=0\end{array}$ & $\begin{array}{l}r^{1}(3 \rightarrow 3) \\
=0.259\end{array}$ & $\begin{array}{l}r^{1}(3 \rightarrow 4) \\
=0.237\end{array}$ & $\begin{array}{l}r^{1}(3 \rightarrow 5) \\
=0.504\end{array}$ \\
\hline & 4 & $\begin{array}{l}r^{1}(4 \rightarrow 1) \\
=0\end{array}$ & $\begin{array}{l}r^{1}(4 \rightarrow 2) \\
=0\end{array}$ & $\begin{array}{l}r^{1}(4 \rightarrow 3) \\
=0\end{array}$ & $\begin{array}{l}r^{1}(4 \rightarrow 4) \\
=0.712\end{array}$ & $\begin{array}{l}r^{m}(4 \rightarrow 5) \\
=0.288\end{array}$ \\
\hline & 5 & $\begin{array}{l}r^{1}(5 \rightarrow 1) \\
=0\end{array}$ & $\begin{array}{l}r^{1}(5 \rightarrow 2) \\
=0\end{array}$ & $\begin{array}{l}r^{m}(5 \rightarrow 3) \\
=0\end{array}$ & $\begin{array}{l}r^{m}(5 \rightarrow 4) \\
=0\end{array}$ & $\begin{array}{l}r^{m}(5 \rightarrow 5) \\
=1\end{array}$ \\
\hline
\end{tabular}

Table 4(b): Mean transition probabilities considering all experts

\begin{tabular}{|c|c|c|c|c|c|c|}
\hline & \multicolumn{6}{|c|}{ States corresponding to respective quality levels (i) } \\
\hline \multirow{2}{*}{$\begin{array}{c}\text { States } \\
\text { corresponding }\end{array}$} & & 1 & 2 & 3 & 4 & 5 \\
\hline & 1 & $\begin{array}{l}p^{1}(1 \rightarrow 1) \\
=0.398\end{array}$ & $\begin{array}{l}p^{1}(1 \rightarrow 2) \\
=0.319\end{array}$ & $\begin{array}{l}p^{1}(1 \rightarrow 3) \\
=0.128\end{array}$ & $\begin{array}{l}p^{1}(1 \rightarrow 4) \\
=0.089\end{array}$ & $\begin{array}{l}p^{1}(1 \rightarrow 5) \\
=0.066\end{array}$ \\
\hline $\begin{array}{l}\text { to respective } \\
\text { quality levels }\end{array}$ & 2 & $\begin{array}{l}p^{1}(2 \rightarrow 1) \\
=0\end{array}$ & $\begin{array}{l}r^{1}(2 \rightarrow 2) \\
=0.269\end{array}$ & $\begin{array}{l}r^{1}(2 \rightarrow 3) \\
=0.327\end{array}$ & $\begin{array}{l}r^{1}(2 \rightarrow 4) \\
=0.198\end{array}$ & $\begin{array}{l}r^{1}(2 \rightarrow 5) \\
=0.206\end{array}$ \\
\hline $\begin{array}{c}\text { quality levels } \\
\text { (i) }\end{array}$ & 3 & $\begin{array}{l}r^{3}(3 \rightarrow 1) \\
=0\end{array}$ & $\begin{array}{l}r^{1}(3 \rightarrow 2) \\
=0\end{array}$ & $\begin{array}{l}r^{1}(3 \rightarrow 3) \\
=0.249\end{array}$ & $\begin{array}{l}r^{1}(3 \rightarrow 4) \\
=0.247\end{array}$ & $\begin{array}{l}r^{1}(3 \rightarrow 5) \\
=0.504\end{array}$ \\
\hline \multirow{2}{*}{ (i) } & 4 & $\begin{array}{l}r^{1}(4 \rightarrow 1) \\
=0\end{array}$ & $\begin{array}{l}r^{1}(4 \rightarrow 2) \\
=0\end{array}$ & $\begin{array}{l}r^{1}(4 \rightarrow 3) \\
=0\end{array}$ & $\begin{array}{l}r^{1}(4 \rightarrow 4) \\
=0.68\end{array}$ & $\begin{array}{l}r^{m}(4 \rightarrow 5) \\
=0.32\end{array}$ \\
\hline & 5 & $\begin{array}{l}r^{1}(5 \rightarrow 1) \\
=0\end{array}$ & $\begin{array}{l}r^{1}(5 \rightarrow 2) \\
=0\end{array}$ & $\begin{array}{l}r^{m}(5 \rightarrow 3) \\
=0\end{array}$ & $\begin{array}{l}r^{m}(5 \rightarrow 4) \\
=0\end{array}$ & $\begin{array}{l}r^{m}(5 \rightarrow 5) \\
=1\end{array}$ \\
\hline
\end{tabular}

Table 5: SQIP's cost coefficients

\begin{tabular}{|c|c|}
\hline$i$ & $C(i)$ \\
\hline 1 & 0 \\
\hline 2 & $\$ 60,000$ \\
\hline 3 & $\$ 125,000$ \\
\hline 4 & $\$ 275,000$ \\
\hline 5 & $\$ 500,000$ \\
\hline
\end{tabular}




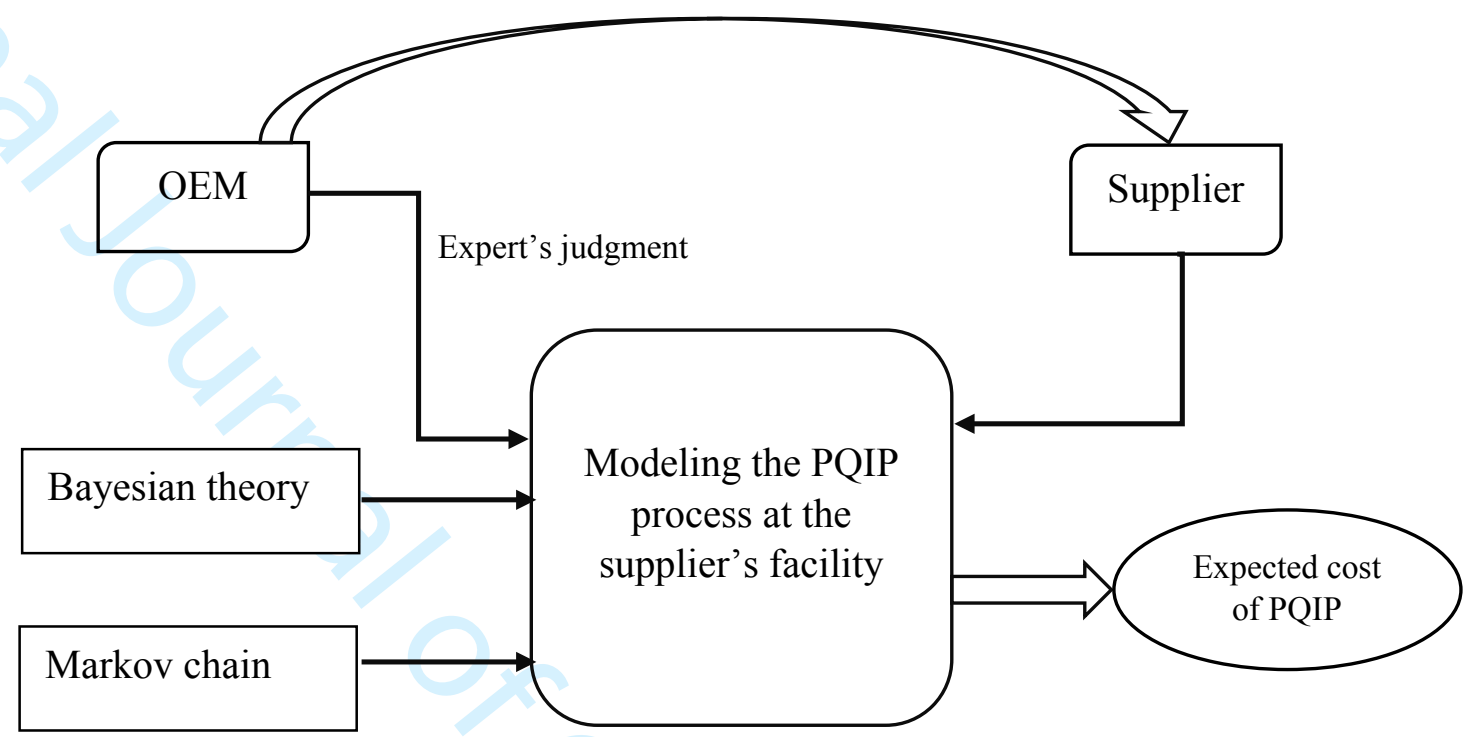

Figure 1: General schema of the research framework

Ascertain the quality levels and populate pairwise component matrices

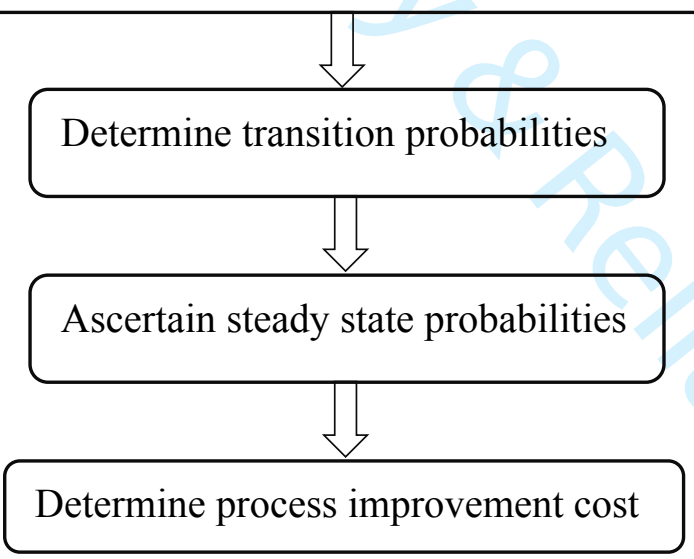

Figure 2: Flowchart for illustration of the framework 